\title{
HOLES IN THE SOLAR CORONA
}

\author{
W. M. GLENCROSS \\ Mullard Space Science Laboratory, Dept. of Physics and Astronomy, \\ University College London, WCIE 6BT, England
}

Summary. Babcock (1961) outlined the sequence of events which takes place in the Sun's atmosphere during a solar cycle. Magnetic field loops, having preferred directions, emerge from the solar surface and thereafter merge with neighbouring loops to produce more extended structures. Although flux tubes emerge with a strong E-W field component, having the field direction reversed from one side of the equator to the other, there is a tendency for the longer loops produced by merging to have a significant N-S alignment (Hansen et al., 1972).

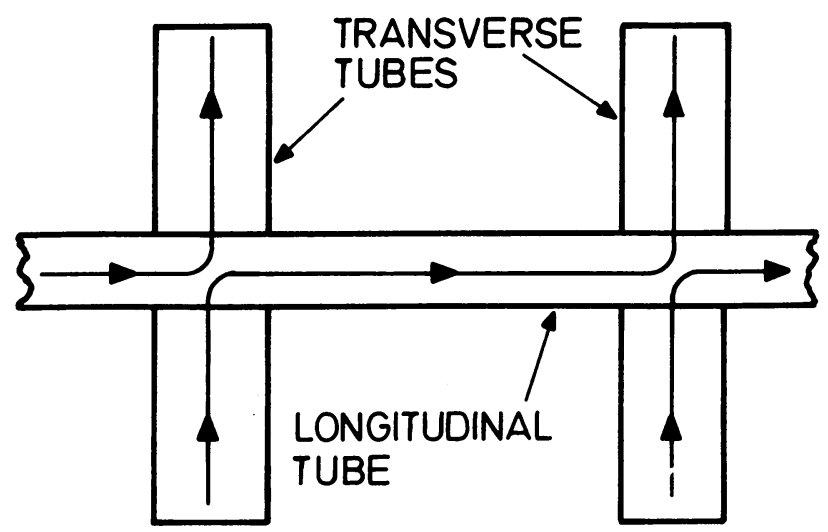

Fig. 1. When each of a pair of parallel flux tubes makes contact with a perpendicular tube, it is possible for field lines (represented as curves with arrow heads) to cross-connect so as to pass from one member of the pair to the other.

Consideration is given here to how the Sun's field might develop once some long flux tubes have been formed with a predominantly N-S alignment. (Such tubes might be part of the general dipole field structure of the Sun). New magnetic arches, having a significant E-W component, emerge from the solar surface and come into contact with the older N-S field so that merging occurs between the approximately perpendicular ropes. Figure 1 shows a plan view along a short length of a N-S aligned flux rope (denoted as the 'longitudinal tube') when two transverse tubes develop beneath it. These latter structures will tend to have parallel fields if they are on the same side of the solar equator. Merging of fields could allow some flux lines to pass between one transverse tube and the other in the manner illustrated.

Figure $2 \mathrm{a}$ shows the situation when the N-S flux tube crosses the solar equator. Because transverse structures on either side of this boundary are anti-parallel, any 
field line linking them has both foot-points on the same side of the longitudinal flux tube. Figure $2 b$ shows a perspective view of the extreme case when the two antiparallel flux tubes have merged completely via the longitudinal rope. In practice there would be cross-connection between numerous transverse structures on one side of the
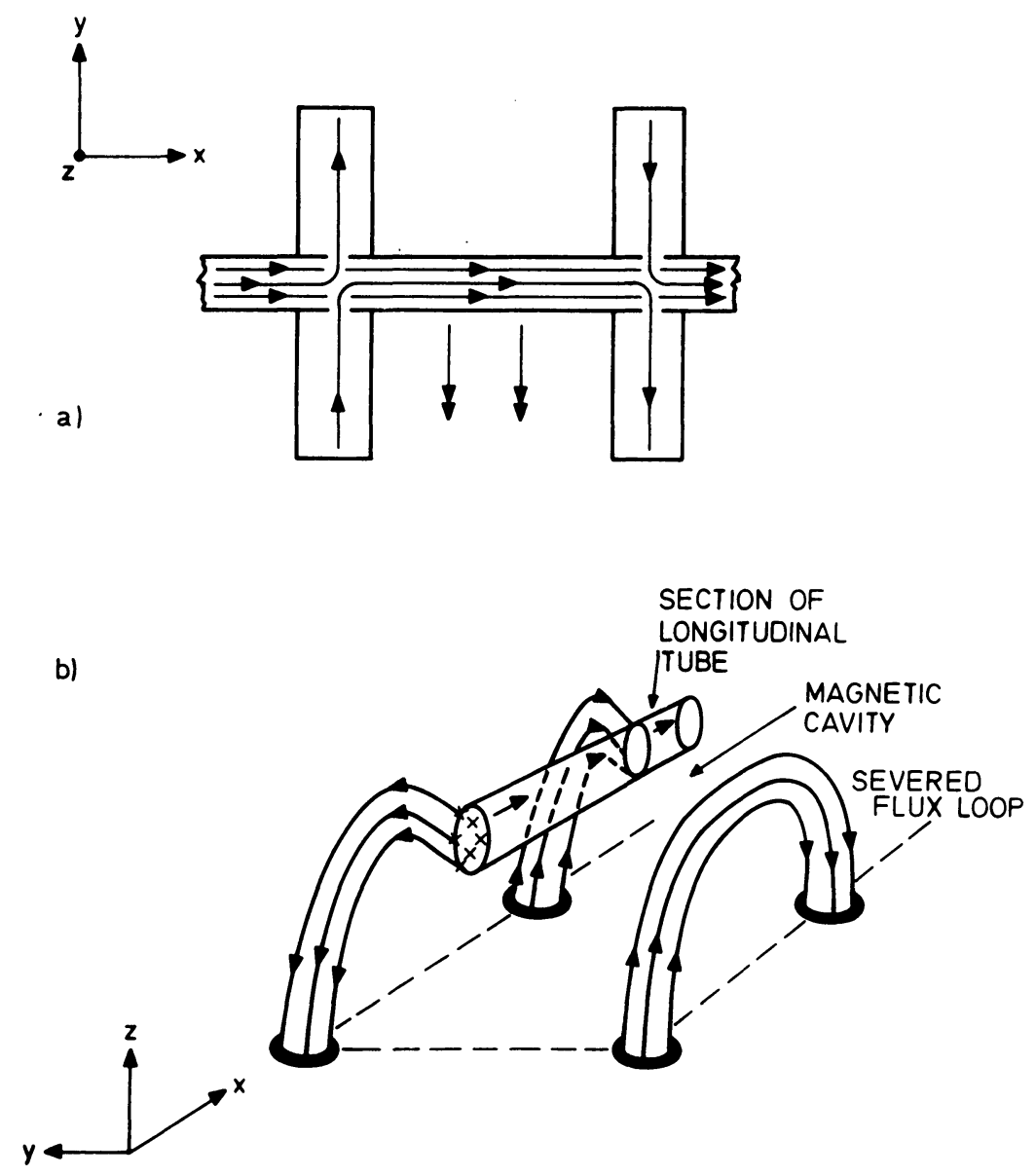

Fig. 2. Diagram (a) is similar to Figure 1, except the transverse arches are now anti-parallel. Diagram (b) is a perspective view of the extreme situation when all field lines from one transverse arch pass into the other.

equator and a similar number on the other side, with the resultant production of a long magnetic cavity.

Conditions shown in Figure 2 can also be expected to form at intermediate latitudes in each hemisphere as a new solar cycle begins to develop. Flux tubes produced here will have polarities which are the reverse of those in the surrounding regions which are still associated with the earlier cycle.

Apart from the coronal holes, it is known that some filaments also have well de- 


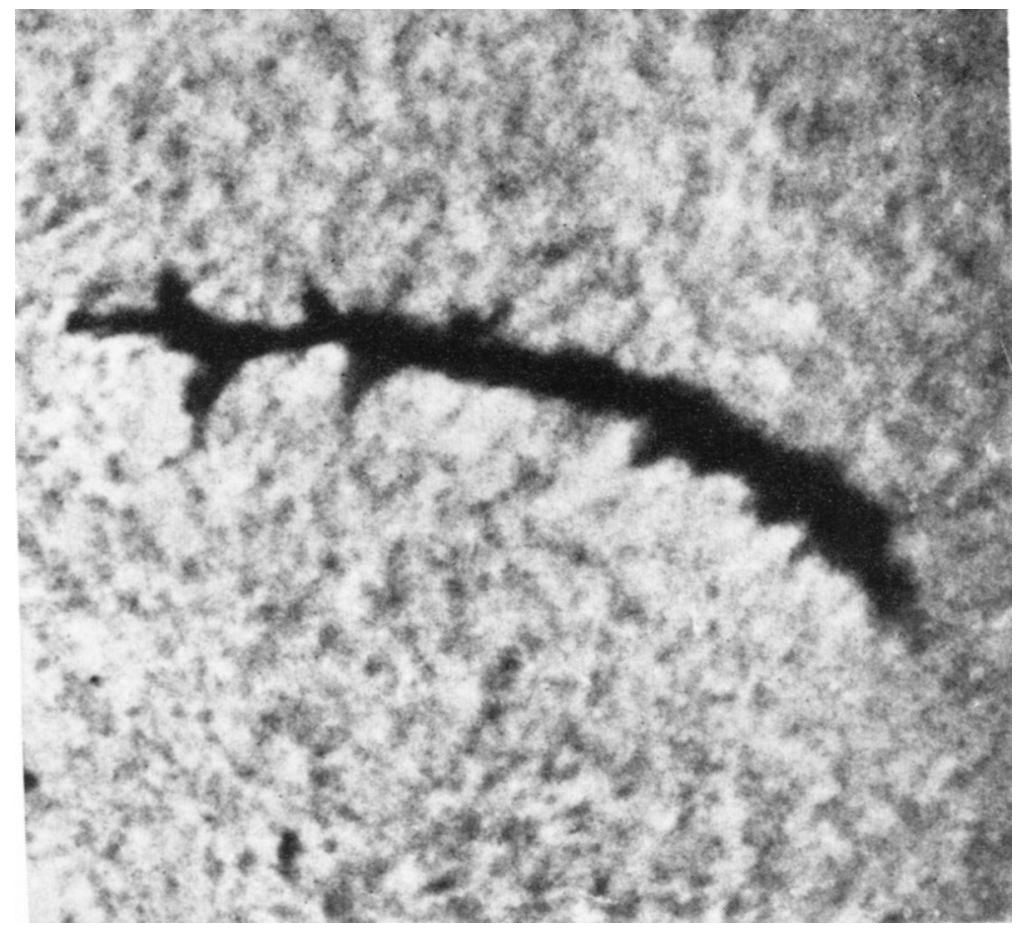

Fig. 3. Observations suggest that supporting magnetic field lines lie perpendicular to the axis of a quiescent prominence, although they are deflected to pass along the filament material itself. Where the filament shown here is viewed from above it seems possible to recognise some flux tubes lying transverse to the axis. If this interpretation is correct, the magnetic structure could be similar to that shown in Figure 1. Prominences formed in favourable locations might be expected to develop the geometry shown in Figure 2, in which case filament cavities might be formed by a mechanism similar to that of coronal holes.

veloped dark cavities associated with them when they are observed in soft X-radiation (Vaiana et al., 1973). There is in fact observational evidence (Ioshpa, 1968) that some prominence structures have magnetic configurations composed of a longitudinal magnetic field, containing condensed material, which is supported by transverse fields along its length. If merging between fields develops, the geometries shown in Figures 1 and $2 \mathrm{a}$ might form. A short length of the filament in Figure 3 appears to show transverse flux ropes, containing condensed material, lying approximately perpendicular to the main line of the prominence.

\section{References}

Babcock, H. W.: 1961, Astrophys. J. 133, 572.

Hansen, S. F., Hansen, R. T., and Garcia, C. J.: 1972, Solar Phys. 26, 202.

Ioshpa, B. A. : 1968, in K. O. Kiepenheuer (ed.), 'Structure and Development of Solar Active Regions', IAU Symp. 35, 261.

Vaiana, G. S., Kreiger, A. S., and Timothy, A. F.: 1973, Solar Phys. 32, 81. 